\section{Einige Störungen der inneren Sekretion bei Pankreaserkrankungen.}

Von Dr. Moritz Cohn, Assistenzarzt der I. Ghirurgischen Abteilung, und Dr. Hans Peiser, Assistenzarzt der I. Internen Abteilung.

Bei den letzten Fällen von Pankreaserkrankungen, die auf der' I. Chirurgischen Abteilung des Rudolf Virchow-Krankenhauses zur Operation kamen, fiel uns eine gewisse Störung in Gesichtsausdruck der Patienten auf. Thr Blick hatte etwas Sticrendes; die Augen waren grol, aber nicht eigentlich vorgetrieben. Die Lidspalten waren auffallend weit. Das Weiß der Skleren machte sich besonders bemerkbar. Beim Blick nach vorn und geradeaus deckte das Oberlid kaum noch den oberen Irisrand. Es waren zwar keine ausgeprägten Glotzaugen, immerhin bestand eine Andeutung von Exophthalmus. Einen Kropf hatte keiner dieser Fälle.

Dieser auffallende Augenbefund wies auf einen Syniptomenkonıplex hin, den wir als Thyreoidismus zu bezeichnen gewohnt sind, und veranlaßte uns, gestützt auf die neueren Arbeiten iiber die innere Sekretion (Biedl, Lüthje) und den Thyreoidismus, unsere Patienten auf das Vorhandensein der iibrigen für den 'Thyreoidismus und die Basedowsche Krankheit charakteristischen Augenphänomene zu prüfen. Hierbei fanden wir in allen Fällen sowohl das Graefesche Symptom sowie das Moebiussche und das Stellwagsche Phänomen in mehr oder minder hohem Grade ausgeprägt.

Die Schilddrüse war in allen Fällen auf Druck empfindlich (Kocher'sches Zeichen).

Mehrfach konnte eine Verbreiterung des Herzens nach links, eine Pulsbeschleunigung sowie eine leichte vasomotorische Erregbarkeit (Dermatographismus) konstatiert werden. Auch die bei einer großen Anzahl von Thyreoidosen (Caro, Kocher) vorhandene relative Lymphozytose suchten wir bei unseren Fällen nachzuweisen; wir fanden dabei Werte von $22-44 \%$ gegen $20-25 \%$ in der Norm - , wenn wir neben den eigent- lichen Lymphozyten noch die großen mononukleären ungranulierten Zellen berücksichtigten.

Des weiteren achteten wir auf die erhöhte Phloridzinglylkosurie. die nach Caro $^{1}$ ) für den Morbus Basedowii und den Thyreoidismus charakteristisch ist; unter geringer Modifikation des von Caro angegebenen Verfahrens gaben wir nach der ïblichen Mittagmahlzeit $11 / 2 \mathrm{cg}$ Phloridzin subkutan. Die Patienten erhielten während der folgenden Stunden bis zur Beendigung des Versuches mäßige Kohlehydratmengen in Form einer Limonade, die $100 \mathrm{~g}$ Himbeersaft enthielt. Der Urin wurde in regelmäßigen Intervallen aufgefangen, gemessen und sein Zuckergehalt in Prozenten und Grammen festgestellt. Der Versuch wurde vorschriftsmäßig erst abgebrochen, wenn mehrere Urinportionen hintereinander zuckerfrei geblieben waren. Während der Gesunde bei dieser Versuchsanordnung insgesant 2-4 g Zucker ausscheidet (cf. Kurve 1), ergibt die

Fräılein Bertha St., 29 Jahre. Normale Schilddtüsenfunktion.

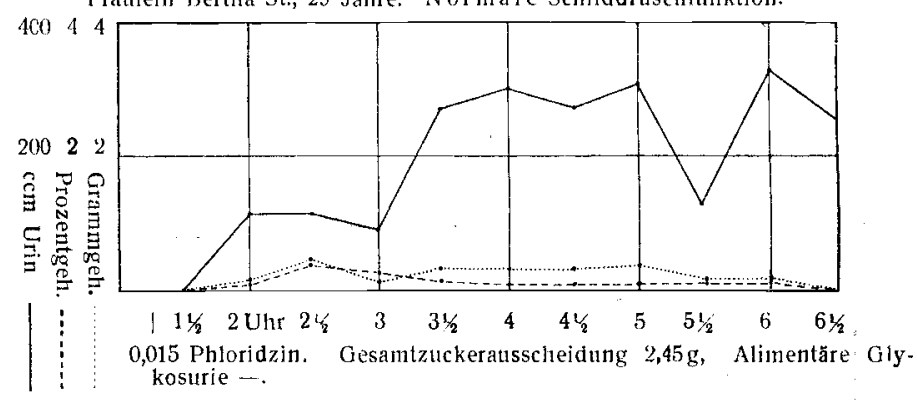

Gesamtzuckerausscheidung beim Hyperthyreoidismus und speziell beim Basedow erhöhte Werte - bis zu $25 \mathrm{~g}$ (cf. Kurve 2); dabei sei besonders hervorgehoben, dals man mit der Beurteilung von Grenzwerten zwischen 5 und $10 \mathrm{~g}$ besonders vorsichtig sein muß.

Aber nicht nur bezïglich der quantitativen Zuckerausscheidung zeigt die Phloridzinglykosurie unter normalen Verhältnissen und bein Thyreoidismus erhebliche Differenzen, sondern auch bezüglich der Art der Ausscheidung bestehen unter den oben genannten Bedingungen erhebliche Unterschiede; stellt man sich die einzelnen Werten entsprechende Urinmenge, Prozent- und Grammgehalt des Urins an Zucker graphisch dar, so zeigt das entstehende Kurvennetz - namentlich die Grammkurve - bei Hypersekretion der Thyreoidea ein ganz charakteristisches (cf. Kurve 2) Verhalten. Unter normalen Bedingungen verläuft die Grammkurve, wie ebenfalls aus Fig. 1 ersichtlich ist, äußerst flach, beim Hyperthyreoidismus dagegen (s. Fig. 2) in einer steilen Zacke, der gelegentlich noch ein bis zwei kleinere Zacken folgen können (vgl. Caros Arbeit).

1) Mitteilungen aus den Grenzgebieten der Medizin und Chirurgie, Bd. $75, \mathrm{H}$. 1 . 
Kurve 2.

Frăulein Dorothea F. 32 Jahre. Basedowsche Krankheit. Struma +, Kocher +, Exophthalmus +, Graefe + , Stellwag + , Moebius + , Tremor + , Dermatograph. + , Cor nach links verbreitert, Puls 108 . Blut: 6200 Leukozyten, 33,2\% Lymphozyten, $1,1 \%$ große Mononukleäre, $63,6 \%$ neutrophile Poly.
nukleäre, $2,1 \%$ eosinophile Polynukleäre.

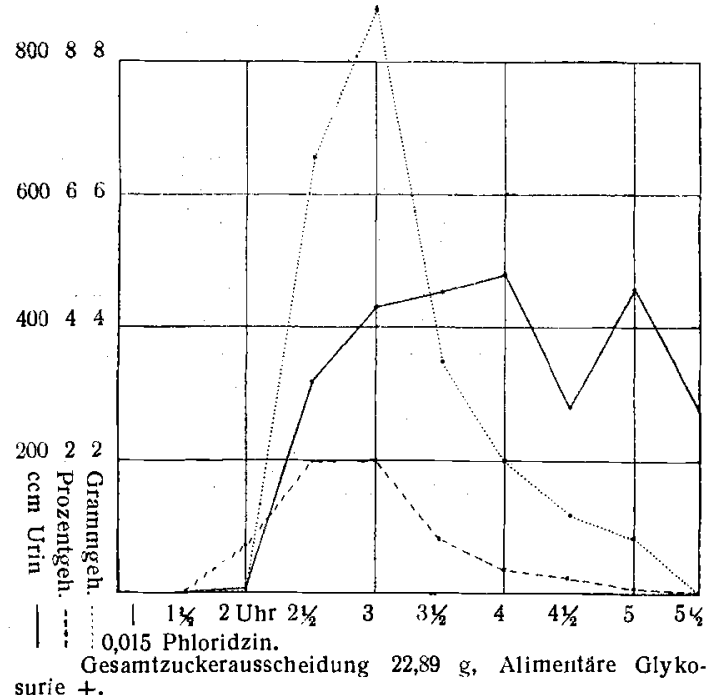

Vergleichen wir die von unseren Pankreaserkrankungen gewonnenen Kurven (3-7) mit den obigen Abbildungen, so fällt eine bemerkenswerte Uebereinstimmung mit der von den Basedowkranken stammenden Kurve auf, die sich nicht bloB auf die Form der Grammkurve, sondern auch auf die Menge der Zuckerausscheidung erstreckt, letztere schwankt in den untersuchten Fällen zwischen $8 \frac{3}{4}$ und $251 / 4 \mathrm{~g}$.

\section{$\mathbf{K}$ u $\mathbf{r}$ ve $\mathbf{3}$.}

Carl H. 44 Jahre. Pancreatitis acuta hämorrhagica. Operiert am 8. Juli 1911. Struma -, Kocher +, Exophthalmus links ganz gering, Graefe links schwach + Stellwag + , Moebius +, Tremor + , Dermatograph. +. Cor o. B., Puls 76, Blut: 6200 nukleäre, 7,4 \% eosinophile Polynukleäre.

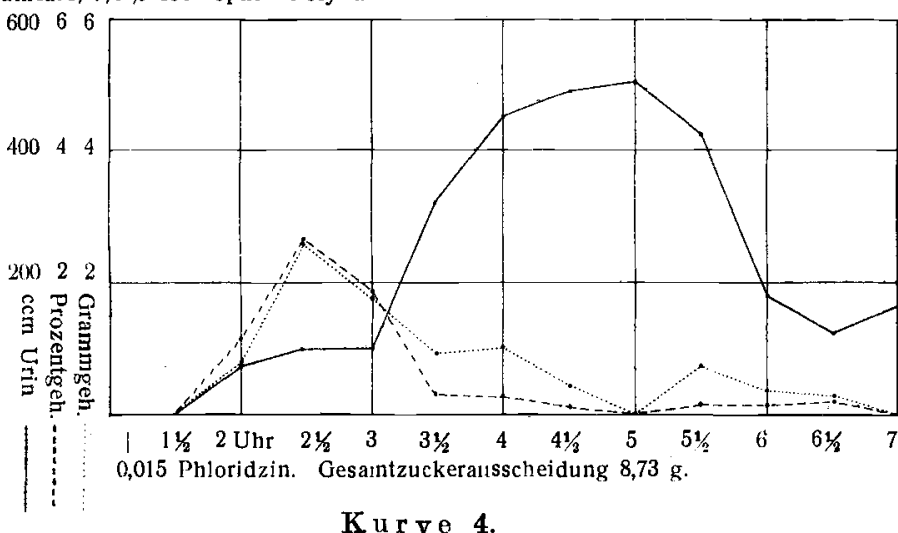

Fr. Hedwig D. 27 Jahre. Pancreatitis acuta haemorrhagica. Operiert am 14. Juli 1911 .

Struma -, Kocher + , Exophthalmus rechts gering, links + , Graefe + , Moebius + . Stellwag + . Tremor + , Derinatographismus + , Cor bis zur Mamillarlinie verbreitert. Puls 120. Blut: 12800 Leukozyten, $36.4 \%$ Lymphozytell, $1,4 \%$ große Mononnkleäre, 59,4 $\%$ neutrophite Polynukleäre. 2,0 \% eosinophile Polynukleäre. $0,8 \%$ Mastzellen.

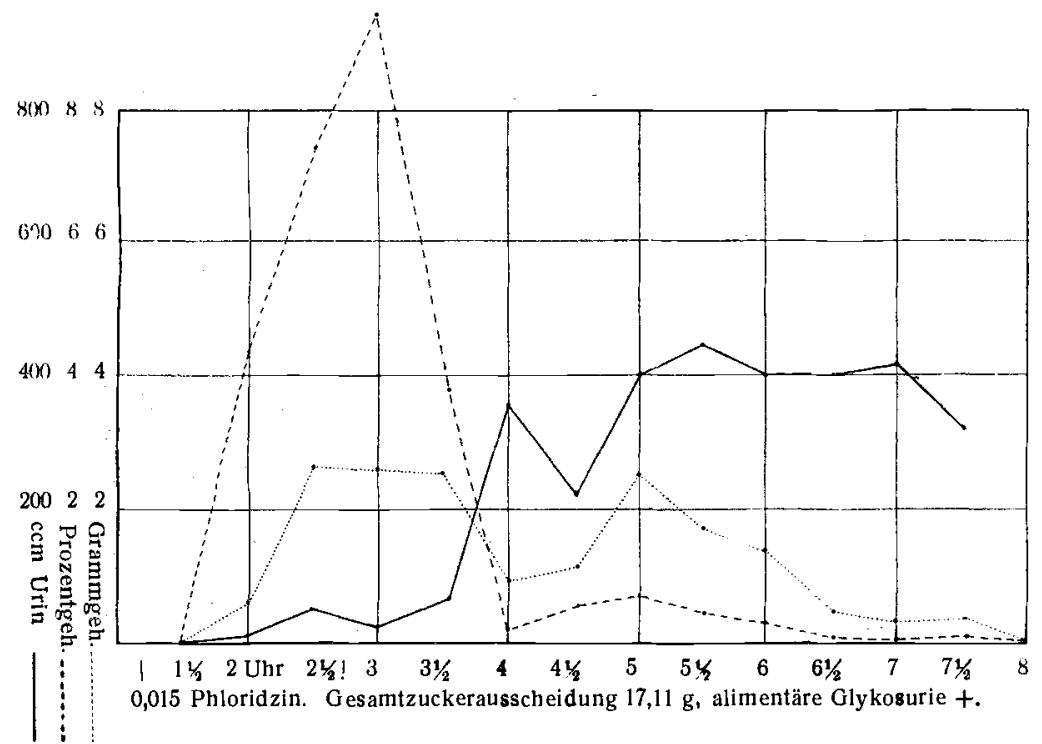

Kur $\forall$ e 5.

Frau Anna K. 62 Jahre. Pancreatitis acuta hämorrhagica. Operiert an 24. Februar 1911 Struma -, Kocher +, Exophthalmus -, Moebius leicht +, Graefe -, Stellwag +, Tremor gering. Cor: Bis zur Mamiltarlinie verbreitert, Puls 80 , Dermatograph. +. Blut: 6500 Leukozyten, $41,3 \%$ Lymphozyten, $0,9 \%$ große Mononukleäre, $55,8 \%$ neutrophile Polynukleăre, $2,0 \%$ eosinophile Polynukleäre.

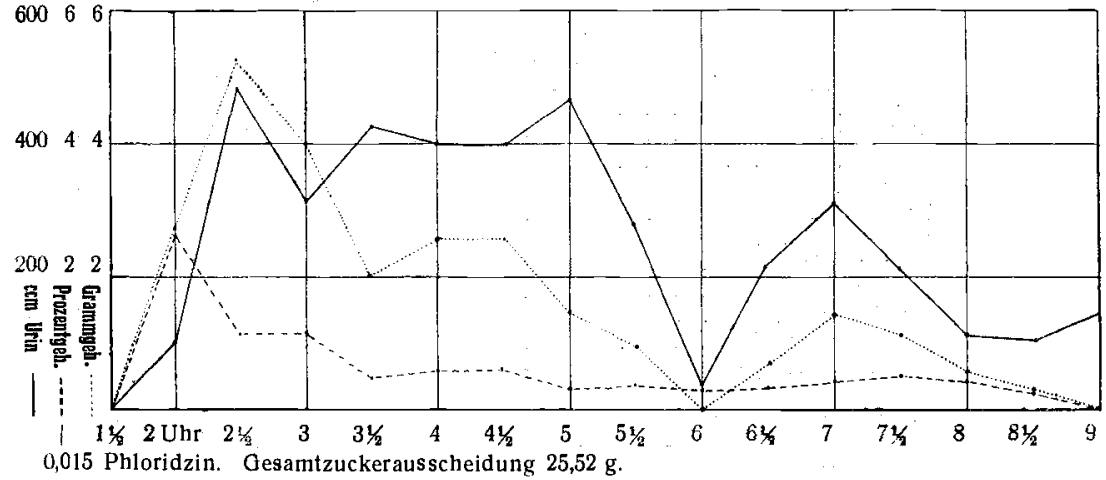

Kurve 6.

Frl. Charlotte S. 21 Jahre. Pancreatitis purilenta mit Pankreasnekrose. Operiert am 14. Jall. 1911 Struma +, Kocher +, Exophthalmus +, Graefe + , Stellwag +, Moebius +, Tremor + Puls 120, Dermatographismus +, Cor: bis zur Mamillarlinie verbreitert. Sehr lange Röhrenknochen. Blut: 6400 Leukozyten. 40,5\% Lymphozyten, 3,6\% große Mononukleäre, 55,0 \%

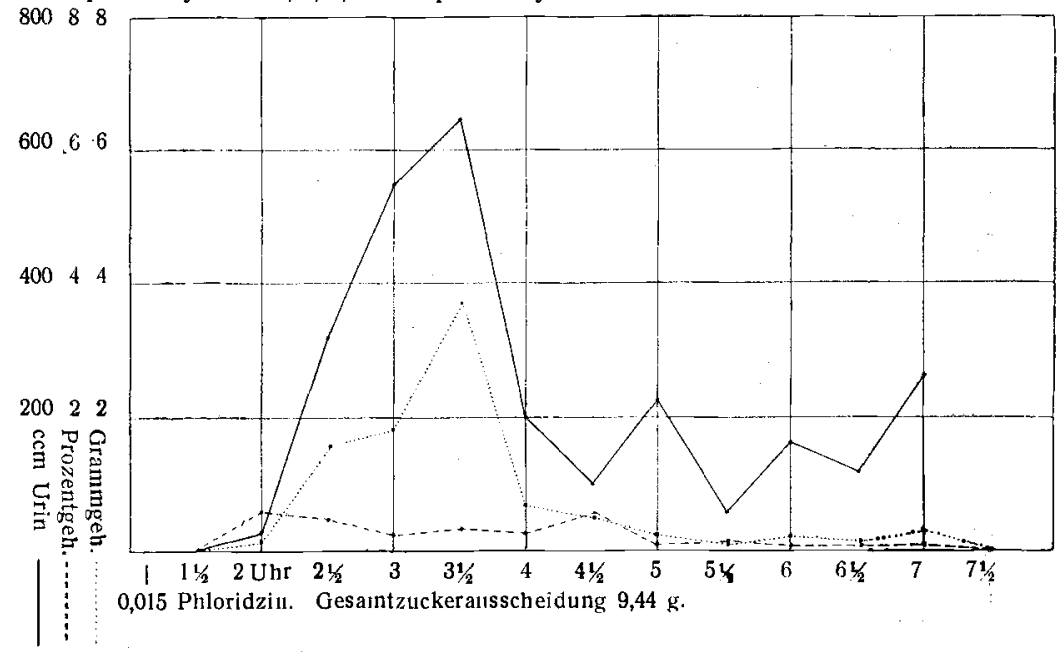

Kurve 7

W1thelm R. 30 Jahre. Pancreatitis chronica interstit. Operiert am 19. Juni. Struma gering. Kocher + , Exophthalmus +, Moebius +, Graefe +, Stellwag +, Tremor + ,
Dermatograph. +, Cor normal, Puls 56. Blut: 7800 Leukozyten, 20,6 $\%$ Lymphozyten, 0,9 $\%$ vroße Mononukleäre, $78,5 \%$ neutrophile Polynukleäre, $0,0 \%$ eosinophile Polynukleäre.

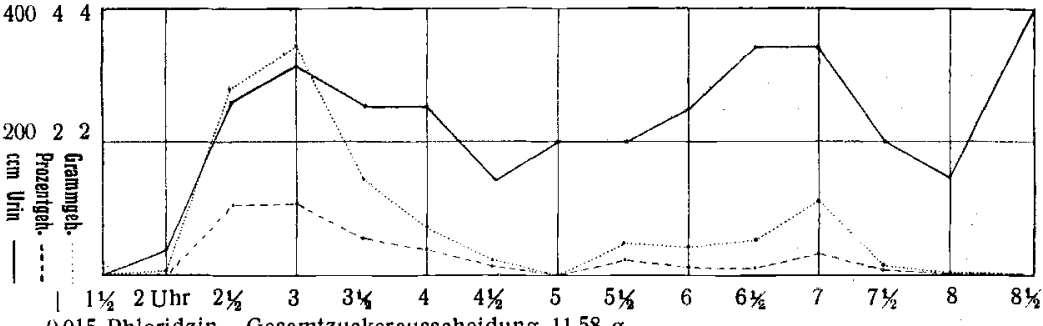

0,015 Phloridzin. Gesamtzuckerausscheidung $11,58 \mathrm{~g}$.

Die Untersuchung auf alimentäre Glykosurie, die Prüfung des Blutes auf seinen Zuckergehalt, der Nachweis von Adrenalin bzw. ähnlich konstituierten Stoffen im Blute und der AdrenalinMydriasis (Löwische Reaktion) mußten aus äußeren Gründen vorläufig unterbleiben.

Stellen wir den Befund der einzelnen Phänomene in unseren fünf zur Untersuchung gekommenen Fällen, bei denen es sich dreimal um Pancreatitis acuta haemorrhagica, einmal um Pancreatitis purulenta und einmal um Pancreatitis chronica interstitialis handelte, übersichtlich zusammen, so ergibt sich folgendes Verhalten:

T a b elle 1.

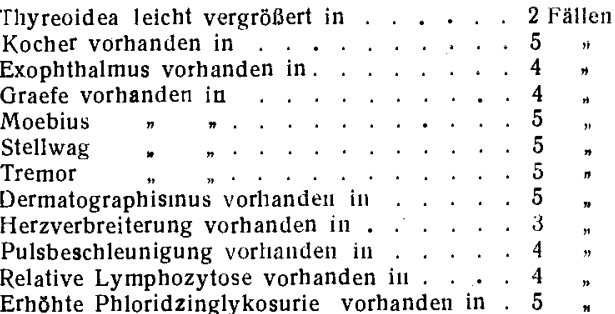


Unter Berücksichtigung der Art der Pankreaserkrankung ergibt sich folgendes Verhalten:

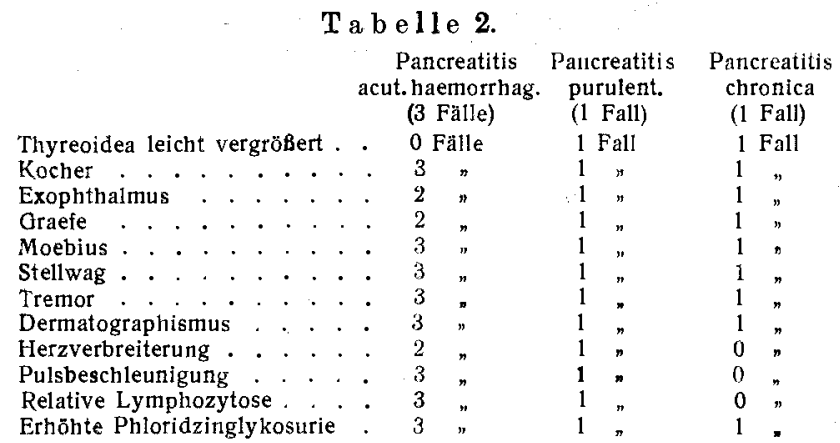

Diese Zusammenstellung unserer Pankreaserkrankungen zeigt eine auffallende Uebereinstimmung in den Resultaten der genannten Untersuchungen. Sie ist um so bemerkenswerter, als sich die eben beschriebenen Phänomene, wie wir uns durch Kontrolluntersuchungen überzeugt haben, bei $\mathrm{Pa}$ tienten, die an Blinddarmentzündungen, Leistenbrüchen etc. operiert waren, nicht fanden.

Wir glauben daher, die eben beschriebenen Symptome mit aller Reserve - mit der Pankreasaffektion in Zusammenhang bringen zu dürfen.

Zum Verständnis unserer Befunde sei darauf hingewiesen, daß das Pankreas und die Nebenschilddrüsen einerseits, die Schilddrüsen und die Nebennieren anderseits hinsichtlich der inneren Sekretion in einem gewissen Antagonismus stehen. Schilddrüse und Nebennieren fördern die Zuckerausscheidung, Pankreas und Nebenschilddrüsen wirken hemmend auf die Zuckerausscheidung ein. Funktionsstörungen des einen Systems bewirken ein Prävalieren des anderen. Theoretisch wäre daher ein Ausfall oder eine Abschwächung der Pankreasgruppe sehr wohl mit einem Ueberwiegen der Schilddrüsengruppe (Hyperthyreoidismus) zu vereinigen. Indessen sind die uns beschäftigenden Fragen aus dem Kapitel der inneren Sekretion zurzeit noch zu wenig gesichert, um unsere Befunde mit absoluter Sicherheit deuten zu können.

Unsere oben besprochenen Beobachtungen bezogen sich auf mehr oder weniger abgelaufene akute und chronische Fälle von Pankreaserkrankungen. Erst in der jüngsten Zeit war es uns möglich, bei einem Falle von frischer Pancreatitis acuta auf die oben genannten Phänomene zu prüfen.

Es handelte sich um eine Frau, die mit der Diagnose Peritonitis in das Krankenhaus kam. Die Patientin machte einen schwer kranken Gesamteindruck, das Gesicht war verfallen, die Augen zurückgesunken, die Lippen blau, Temperatur $39^{\circ}$, Puls 140 , kaum fühlbar. Im oberen Bauche fühlte man eine ungewisse, querverlaufende Resistenz in Nabel. höhe, ein Punkt stärkster Druckempfindlichkeit lag an der Grenze von unterem und mittlerem Drittel der Nabel-Schwertfortsatzlinie. Die Blinddarmgegend war druckempfindlich - aber nicht so stark wie die Magengegend; die Bauchdecken waren im übrigen leicht gespannt. Die Leukozytose betrug 20000 , die relative Lymphozytose über $20 \%$; (diese Zahl ist aber höher zu bewerten, weil die relative Lymphozytose bei entzündlichen Bauchaffektionen durch einfache neutrophile Leukozytose zum Teil verdeckt ist.)

Das klinische Bild ließ in diesem Falle an die Möglichkeit einer Pankreatitis denken; bestärkt wurden wir in dieser Annahme durch den Nachweis des Möbiusschen Phänomens und durch den Befund der relativen Lymphozytose. Die Diagnose wurde deswegen auf Pancreatitis acuta haemorrhagica gestellt und durch den Operationsbefund bestätigt.

Wenn auch in diesem Falle von frischer akuter Pankreatitis Augenphänomene und eine relative Lymphozytose vorhanden war, so dürfen wir doch kaum annehmen, daß diese Befunde in Jedem Falle von akuter Pankreatitis zu erwarten sind.

Aber auch bei positivem Augenbefunde ist Vorsicht in der Deutung desselben dringend geboten. Besteht bei einer Peritonitis mit klinisch unbekannten Ausgangspunkten der Exophthalmus schon seit langer Zeit, so muß man bei der Bewertung dieses Exophthalmus und der übrigen Augenphänomene sehr vorsichtig sein. Einen derartigen Fall haben wir in den letzten Tagen beobachtet.

Es handelte sich um eine Frau, die 21/2 Tag vor ihrer Aufnahme in das Krankenhaus plötzlich mit heftigen Leibschmerzen und Erbreehen erkrankt war. Die Schmerzen dauerten an und lokalisierten sich hauptsächlich in der Oberbauchgegend. Stuhl und Winde setzten seit Beginn der Krankheit aus. Die Frau hatte mehrere Jahre in Brasilien gelebt und an,,Blutdiarrhöe" gelitten. $\mathrm{Ab}$ und zu waren früher ziehende Schmerzen in Leib aufgetreten. Seit langen Jahren hat die Frau ,vorstehende Augen."

Die Patientin machte keinen ausgesprochen schwerkranken Gesamt. eindruck. Es bestand ein Exophthalmus mäBigen Grades, Konvergenzschwäche des rechten Auges. Zunge belegt. Temperatur 39º Puls 110. Leukozyten 19 000, Lymphozyten $15 \%$.

Der Bauch war in allen Teilen mehr oder weniger gespannt und schmerzhaft. Die größte Druckempfindlichkeit bestand in der Oberbauchgegend, besonders in der Mittellinie oberhalb des Nabels. Eine Resistenz war nirgends zu fühlen. Darmsteifungen waren nicht zı sehen.

Die klinische Diagnose lautete: Bauchfellentzündung unbekannter Ursache. Eine Blinddarmentzündung oder ein perforiertes Magengeschwïr ist mit großer Wahrscheinlichkeit auszuschließen. Mit einer gewissen Berechtigung konnte man daher an die Möglichkeit einer akuten Pankreatitis denken. Obwohl das relativ gute Aussehen der Patientin und der Puls eigentlich nicht zu dem klinischen Bilde einer akuten Pankreatitis vom dritten Tage paßten, nahmen wir der Augenphänomene wegen eine akute Pankreatitis an - bis wir uns in Narkose von dem Vorhandensein eines faustgroßen, beweglichen, dreifingerbreit unter dem Nabel gelegenen Tumors überzeugten. Dieser Tumor war vorher nicht fühlbar gewesen. Das klinische Bild erschien nun in einem ganz anderen Iichte. Die Diagnose ,innere Einklemmung" lag nahe und wurde durch die Operation bestätigt.

In diesem Falle bestand eine Forme fruste des Basedow, die uns beinahe irregeführt hätte.

Obwohl die Untersuchungsergebnisse in allen unseren Fällen von Pankreaserkrankungen untereinander übereinstimmen, sind wir weit entfernt, solange wir uns nicht auf ein größeres Beobachtungsmaterial stützen können, irgendwelche Schlüsse hinsichtlich der Diagnostik ziehen zu wollen. Wir wollen nicht einmal behaupten, daß ein Zusammenhang zwischen der Pankreaserkrankung und den Erscheinungen besteht, die man als Thyreoidismus zu bezeichnen gewohnt ist, obwohl theoretisch ein derartiger Zusammenhang möglich wäre.

Wir glauben aber weitere Kreise auf unsere Beobachtungen aufmerksam machen zu dürfen und eine Nachprüfung obiger Phänomene anregen zu sollen.

Zusammenfassung. Bei fünf von uns untersuchten Pankreaserkrankungen - drei Fälle von Pancreatitis acuta haemorrhagica, je ein Fall von Pancreatitis purulenta und Pancreatitis chronica interstitialis - fanden sich folgende auf Störung der inneren Sekretion hindeutende Symptome: Exophthalmus - mehr oder weniger - in vier Fällen, Graefesches Phänomen in vier Fällen, Moebiussches und Stellwagsches Zeichen in fünf Fällen, Tremor und Dermatographismus in fünf Fällen, relative Lymphozytose in vier Fällen, erhöhte Phloridzinglykosurie in fünf́ Fällen, Kochersches Zeichen in fünf Fällen. Die Zahl der bisher untersuchten Fälle ist indes noch zu gering, um irgendwelche Schlüsse ziehen zu können. 